\title{
Altered Intrinsic and Casual Functional Connectivities of the Middle Temporal Visual Motion Area Subregions in Chess Experts
}

\section{OPEN ACCESS}

Edited by:

Jiaojian Wang,

University of Electronic Science and Technology of China, China

Reviewed by:

Jinping $X u$

Shenzhen Institutes of Advanced

Technology, Chinese Academy of Sciences, China

Qinmu Peng,

Huazhong University of Science and Technology, China

*Correspondence:

Peng Dong

dongpeng@wfmc.edu.cn

Specialty section:

This article was submitted to Brain Imaging Methods, a section of the journal

Frontiers in Neuroscience

Received: 14 September 2020

Accepted: 28 October 2020

Published: 01 December 2020

Citation:

Song L, Ge Y, Long J and Dong P (2020) Altered Intrinsic and Casual

Functional Connectivities of the Middle Temporal Visual Motion Area

Subregions in Chess Experts.

Front. Neurosci. 14:605986.

doi: 10.3389/fnins.2020.605986

\author{
Limei Song ${ }^{1}$, Yanming Ge ${ }^{2}$, Jinfeng Long ${ }^{1}$ and Peng Dong ${ }^{1 *}$ \\ ${ }^{1}$ School of Medical Imaging, Weifang Medical University, Weifang, China, ${ }^{2}$ Medical Imaging Center, Affiliated Hospital \\ of Weifang Medical University, Weifang, China
}

An outstanding chess player needs to accumulate massive visual and spatial information for chess configurations. Visual motion area (MT) is considered as a brain region specialized for visual motion perception and visuospatial attention processing. However, how long-term chess training shapes the functional connectivity patterns of MT, especially its functional subregions, has rarely been investigated. In our study, using resting-state functional connectivity (RSFC) and Granger causality analysis (GCA), we studied the changed functional couplings of MT subregions between 28 chess master players and 27 gender- and age-matched healthy novices to reveal the neural basis of long-term professional chess training. RSFC analysis identified decreased functional connections between right dorsal-anterior subregion (Cl1.R) and left angular gyrus, and increased functional connections between right ventral-anterior MT subregion (Cl2.R) and right superior temporal gyrus in chess experts. Moreover, GCA analyses further found increased mutual interactions of left angular gyrus and Cl1.R in chess experts compared to novice players. These findings demonstrate that long-term professional chess training could enhance spatial perception and reconfiguration and semantic processing efficiency for superior performance.

\footnotetext{
Keywords: visuospatial attention network, resting-state functional connectivity, chess experts, visual motion area, granger causality analysis
}

\section{INTRODUCTION}

Chess playing is a complex intellectual game that is considered as a hard mental activity that requires sophisticated problem-solving skills. During chess playing, many kinds of cognitive processes are involved, e.g., working memory, attention, visuospatial perception, motivation, and decision making (Onofrj et al., 1995; Amidzic et al., 2001; van der Maas and Wagenmakers, 2005). The daily skill practice can result in the representative neural structural and functional changes that underlie the related particular skill (Maguire et al., 2000; Gaser and Schlaug, 2003a,b; Draganski et al., 2004; Zou et al., 2012; Song et al., 2020). Chess is seen as a typical example for an expertise task requiring domain-specific experience to study brain structural and functional plasticity. Therefore, to reveal the functional changes, master chess players provide a good opportunity to uncover the neural basis of brain plasticity induced by long-term skill practice (Zhou et al., 2018). 
Chess players acquire outer information such as chess objects and position information on the board mainly relying on visual inputs. To become an outstanding chess player, one needs to accumulate massive visual experience of chess configurations. This experience confers distinct advantages in chess experts when encountering situations that commonly appear in games compared with novices. Increasing evidence has demonstrated that chess expertise can result in changed interactions between different brain networks due to frequent practice (Bilalic et al., 2011; Krawczyk et al., 2011; Duan et al., 2012a,b, 2014; Song et al., 2020), especially visuospatial attention network, which includes dorsal and ventral attention networks playing a key role in preparing, applying goal-directed selection for stimuli and responses, and detecting the salient targets (Corbetta and Shulman, 2002; Astafiev et al., 2004; Kincade et al., 2005; Fox et al., 2006; Wang et al., 2016; Wu et al., 2016). Visual motion area (MT), which is traditionally considered to be specialized for the perception of motion in the visual modality, plays an important role in visual motion and visuospatial attention processing and is the hub of the visuospatial attention network (Gao et al., 2020). Recently, based on meta-analysis in BrainMap database and task-related coactivation-based parcellation approach, Gao et al. (2020) identified the MT region and parcellate this area into several different functional subregions showing different coactivation patterns and functions. Revealing the specific changes of MT subregions with a fine-grained MT atlas in master chess players compared to novices could provide a new avenue to delineate the neural basis of long-term skill practice on brain plasticity.

In this study, we studied the specific changes of functional connectivity patterns of each MT subregion in chess experts to uncover the neuroanatomical basis of brain plasticity induced by long-term chess practice using a full data-driven approach. First, bilateral MT subregions were defined with a fine-grained MT atlas (Gao et al., 2020), and the whole-brain resting-state functional connectivity (RSFC) analysis for each subregion was performed. Next, Granger causality analysis (GCA) was used to identify the causal interactions between MT subregions and regions showing changed RSFC. Finally, correlation analyses were done to reveal the associations between changed RSFC or GCA and behavioral performances.

\section{MATERIALS AND METHODS}

\section{Participants}

Twenty-eight chess players (female/male $=10 / 18$, mean \pm standard deviation of age $=27.64 \pm 9.15$ years; mean \pm standard deviation of education $=13.43 \pm 2.71$ years $)$ and 27 novice players (female/male $=15 / 12$, mean \pm standard deviation of age $=26.37 \pm 6.68$ years; mean \pm standard deviation of education $=14.24 \pm 3.06$ years) were used in this study. The data were accessed from the "1000 Functional Connectomes Project"1. The professional chess players had been

${ }^{1}$ http://fcon_1000.projects.nitrc.org/indi/pro/wchsu_li_index.html
TABLE 1 | Demographics of chess and novice players used in the present study.

\begin{tabular}{lccc}
\hline & $\begin{array}{c}\text { Chess players } \\
(\boldsymbol{n}=\mathbf{2 8})\end{array}$ & $\begin{array}{c}\text { Novice players } \\
(\boldsymbol{n}=\mathbf{2 7})\end{array}$ & $\boldsymbol{p}$ value \\
\hline Gender (male/female) & $18 / 10$ & $12 / 15$ & 0.14 \\
Age (years) (mean \pm SD) & $27.64 \pm 9.15$ & $26.37 \pm 6.68$ & 0.57 \\
Education (years) (mean \pm SD) & $13.43 \pm 2.71$ & $14.24 \pm 3.06$ & 0.31 \\
FD_power (mean \pm SD) & $0.26 \pm 0.25$ & $0.21 \pm 0.07$ & 0.33 \\
\hline
\end{tabular}

A Pearson $\chi^{2}$ test was used for gender comparison. Two-sample t tests were used for age, education, and FD values comparisons. FD, frame displacement.

seriously training regularly (training time: $4.17 \pm 1.72 \mathrm{~h} /$ day), whereas the 27 gender-, age-, and education-matched (gender: $p=0.14$; age: $p=0.57$; education: $p=0.31$ ) novice players understand the rules and simple strategies only for playing Chinese chess. All the sample characteristics are listed in Table 1. No differences on observation skills or clear-thinking ability were found between these two groups (Li et al., 2015). All participants were right-handed and had no history of psychiatric or neurological disorders. Written informed consent was obtained from each subject, and approval was obtained through the local institutional review board of the West China Hospital of Sichuan University. The detailed information of this dataset can be found in $\mathrm{Li}$ and colleagues' study (Li et al., 2015).

\section{Resting-State Functional Magnetic Resonance Imaging Data Acquisition}

The resting-state functional magnetic resonance imaging (fMRI) data were acquired for each subject using a Siemens 3.0$\mathrm{T}$ MRI scanner at the MR Research Center of West China Hospital of Sichuan University, Chengdu, China. All MR procedures were performed when subjects were relaxed with their eyes open and fixated on a cross-hair centered on the screen. A T2-weighted gradient-echo echo-planar imaging (EPI) sequence was used to collect the resting-state fMRI scans. A total of 205 whole-brain echo-planar pulse sequence volumes were acquired using the following parameters: repetition time $=2,000 \mathrm{~ms}$; echo time $=30 \mathrm{~ms}$; flip angle $=90^{\circ}$; axial slice thickness $=5 \mathrm{~mm}$, with no gap; slice number $=30$; and voxel size $=3.75 \times 3.75 \times 5 \mathrm{~mm}^{3}$.

\section{Resting-State fMRI Data Preprocessing}

The resting-state fMRI scans were preprocessed including the following steps. The first 10 volumes were discarded to facilitate magnetization equilibrium, and the remaining images were realigned to the first volume to correct head motion. Next, all fMRI scans were normalized to the Montreal Neurological Institute EPI template and resampled to $3 \times 3 \times 3 \mathrm{~mm}^{3}$. The fMRI scans were smoothed using a Gaussian kernel of 6-mm full width at half maximum. Friston-24 head motion parameters, white matter, cerebrospinal fluid, and global mean signals were then regressed out. Finally, the functional images were filtered with a temporal band path of 0.01 to $0.1 \mathrm{~Hz}$. To further exclude the head motion effects on functional connectivity analysis, a scrubbing method was conducted to censor each subject's bad 


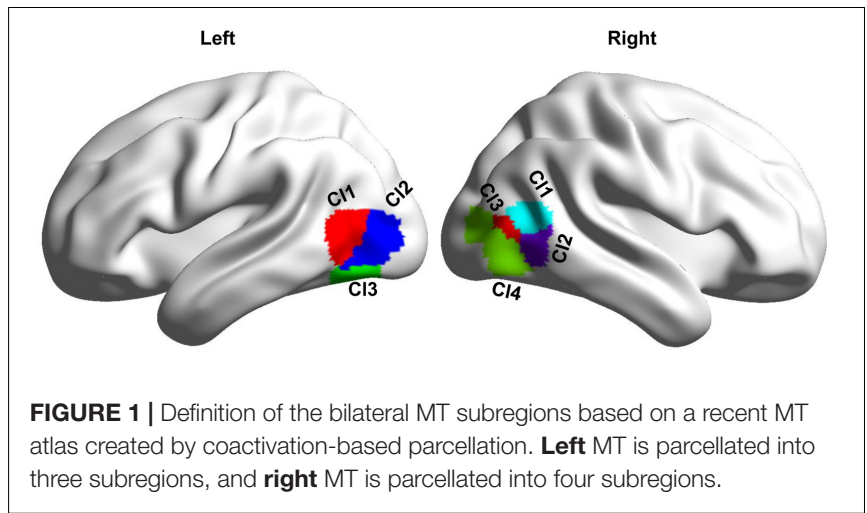

fMRI scans to find out the mean frame displacement, which was above $0.5 \mathrm{~mm}$, and one volume before and two volumes after the bad volume were discarded (Power et al., 2012). Only the participants with the fMRI scans more than half of the total time points were kept for the following analyses. There is no significant difference in head motion between these two groups $(p=0.33$; Table 1).

\section{Definition of the MT Subregions}

MT subregions were defined with an MT atlas which, was created using coactivation-based parcellation approach (Gao et al., 2020). In this atlas, the left MT was parcellated into three subregions including two dorsal subregions $(\mathrm{Cl} 1$ and $\mathrm{Cl} 2)$ and one ventral subregion $(\mathrm{Cl} 3)$, and the right MT was parcellated into four subregions including two anterior subregions ( $\mathrm{Cl} 1$ and $\mathrm{Cl} 2)$, one middle subregion $(\mathrm{Cl} 3)$, and an additional posterior subregion (Cl4) (Gao et al., 2020; Figure 1).

\section{Functional Connectivity Analyses}

To explore whether the whole-brain functional connectivity showed differences in the MT subregions between chess and novice players, a whole-brain functional connectivity map defined by the Pearson correlation of each MT subregion for each subject was calculated. To improve normality, Fisher's $z$ transformation was applied to change the $r$ values to $z$ values. To identify the functional connectivity pattern differences between chess experts and novices, two-sample $t$ tests were performed in a voxel-wise manner, and the significant level was determined with a cluster-level Monte Carlo simulation-corrected threshold of $p<0.05$ (cluster-forming threshold at voxel-level $p<0.001$ ).

\section{GCA for Chess and Novice Players}

Granger causality analysis was performed to further explore the causal interaction of brain areas with changed RSFC between chess and novice players. The mean time series of these brain areas were extracted, and the bivariate GCA was performed on the time series to test the causal interactions. The order of the autoregressive model used for computation of the influence measure was selected using the Bayesian information criterion, and magnitudes of causal interactions were obtained for each subject (Wang et al., 2017a, 2019). GCA was performed using the Causal Connectivity Toolbox (Seth, 2010). Two-sample $t$ tests were performed on the magnitudes of causal interactions to identify the significant causal influences between brain areas using a significant threshold of $p<0.05$ corrected by Bonferroni correction.

\section{Correlation Analysis}

To explore the relationship between changed resting-state functional or causal connectivities and the amount of time that chess players spent on professional training, correlation analyses were performed, and the significant level was set at $p<0.05$.

\section{RESULTS}

\section{Differences in Functional Connectivities of MT Subregions}

Whole-brain RSFC analyses identified only significant differences of right MT subregions in chess players. The right Cl1 (CI1.R) showed significantly reduced functional connectivity with left angular gyrus (AG.L) in chess players compared with novices (Figure 2). A significantly increased functional connectivity between right $\mathrm{Cl} 2$ (CI2.R) and right superior temporal gyrus (STG.R) was found in chess experts (Figure 2).

\section{Differences in Casual Interactions}

To further determine the differences in causal interactions, the GCA was performed and identified a significantly increased magnitude of mutual causal interactions between Cl1.R and AG.L in chess players compared with novices (Figure 3). Chess players showed no significant differences in magnitude of causal interactions between Cl2.R and STG.R (Figure 3).

\section{Correlation Analyses}

Correlation analyses did not find significant associations between the functional and effective connectivity strength and the

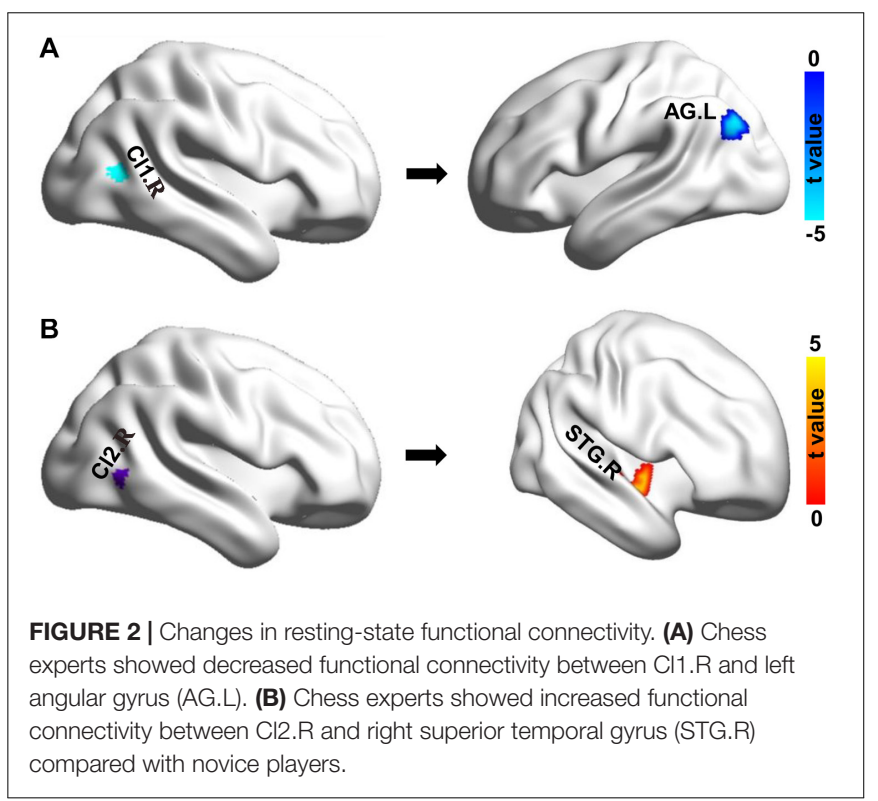




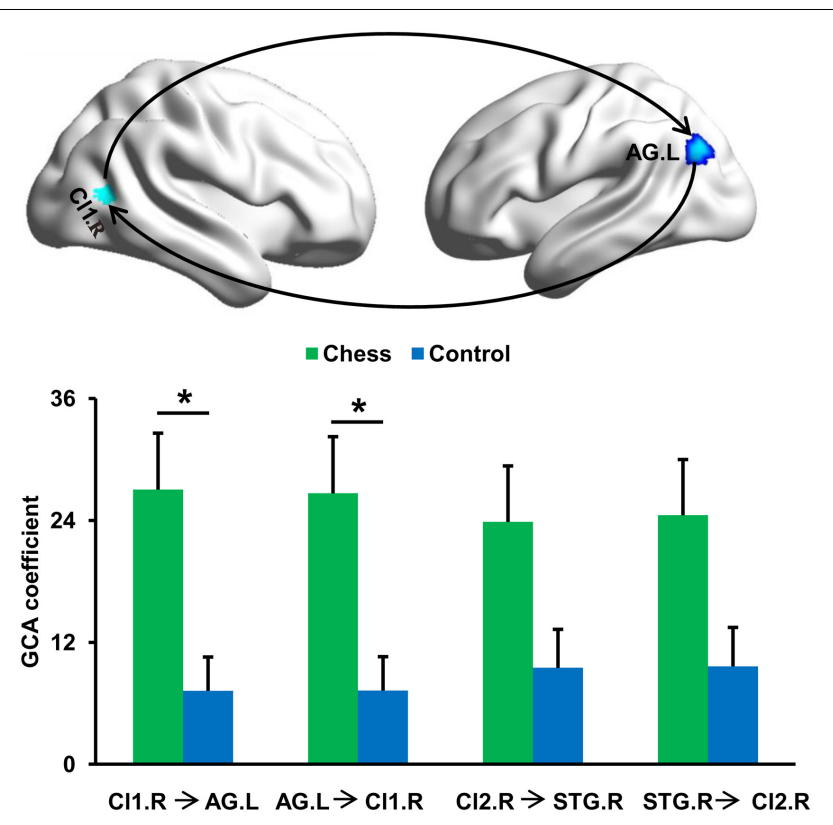

FIGURE 3 | Changes in effective connectivities. Chess experts showed increased mutual effective connectivity between Cl1.R and AG.L compared with novice players. No significant difference in effective connectivity between Cl2.R and STG.R in chess experts was found compared with novice players. '*' represents the significant difference.

amount of time chess players spending on professional training (all $p>0.05$ ).

\section{DISCUSSION}

In the present study, using resting-state functional MRI, we revealed that specifically long-term chess training can change the functional interactions of MT subregions. Connectivity analyses identified significantly increased RSFC of Cl1.R and Cl2.R in chess experts compared with novices. Specifically, decreased functional connectivity between Cl1.R and AG.L and increased functional connectivity between Cl2.R and STG.R were found in chess experts. In addition, GCA analysis further revealed chess experts showed enhanced mutual interactions between Cl1.R and AG.L. These findings suggest chess visual practice may enhance the neuroplasticity of Cl1.R and Cl2.R in MT, and the increased functional connections resulting from the professional chess training may enhance visuospatial attention and semantic and auditory memory processing.

MT is approximately located in the junction of the posterior middle temporal gyrus and occipital gyrus (Smith et al., 2006). Increasing evidence demonstrates that $\mathrm{MT}$ is a functional complex brain area with several distinct functional subregions (Smith et al., 2006; Kolster et al., 2010). Recently, using metaanalysis of visual motion task and coactivation-based parcellation approach with BrainMap database, Gao and colleagues identified the bilateral MT in human and subdivided this area into several different functional subregions (Gao et al., 2020). By comparing the whole-brain functional connectivity maps, we demonstrate that long-term professional chess training could induce the functional plasticity of different subregions. Moreover, neurons in MT are highly sensitive to the speed and direction of visual stimuli in motion (Albright, 1984). Previous studies have revealed that daily chess practice could activate this area during chessrelated visual search tasks for processing visually presented chess game positions (Bilalic et al., 2010, 2011) and could change the RSFC in a behaviorally specific manner (Hampson et al., 2006; Lewis et al., 2009). In our study, we found that long-term chess practice can result in significantly changed RSFC patterns of MT in chess experts. The changed functional connections in MT subregions suggest Cl1.R and Cl2.R are the critical hub regions for integrating visual information processing in chess experts.

Chess experts showed enhanced functional connectivity between Cl2.R and STG.R. STG is an association cortex and plays a key role in auditory memory (Rajarethinam et al., 2000; Zevin, 2009; Wang et al., 2015). Auditory memory involves auditory reception of orally presented information, auditory processing and storing, and finally recalling what has been heard. A previous study reported that auditory memory pathways involved during the chess game can be affected by the process of long-term chess playing (Fattahi et al., 2015). Fattahi et al. (2015) found that chess experts had better auditory memory function than nonchess players. Besides, several studies have demonstrated MT region is also related to motion of auditory (Poirier et al., 2006; Watkins et al., 2013). The increased functional couplings between Cl2.R and STG.R suggest long-term chess practice could strength auditory memory to advance better performances during the chess game. In addition, several studies showed that STG is implicated in the processing of eye movements, as well as in the visual analysis of social information conveyed by gaze and body movement (Allison et al., 2000; Hoffman and Haxby, 2000; Pelphrey et al., 2005). The long-term and intensive visual practice of chess expertise may elevate functional interactions between Cl2.R and STG.R in chess experts for excellent concentration in devoting to the game.

We also found decreased functional connectivity but increased mutual effective connectivities between Cl1.R and AG.L in chess players. AG.L is primarily involved in reading and semantic processing (Wang et al., 2017b). Previous studies about reading also revealed that AG.L is involved in orthography to semantic mapping (Horwitz et al., 1998). Thus, the decreased functional connectivity between AG.L and Cl1.R may be related to higher efficiency and less effort for semantic processing related to chess game. This conclusion is supported by the increased mutual effective connectivities between these two areas. The increased effective connectivities between Cl1.R and AG.L indicate faster button-up and top-down information communication in chess experts than in novices.

There are several limitations to the present study. First, the number of the included chess players is small. A larger sample is needed to further validate the current findings. Second, our study focused only on resting-state functional connections. A topological approach of structural brain connectivity networks should be explored in future studies to reveal the anatomical basis of any functional changes. Third, as the technical limitation of fMRI acquisition, the GCA of fMRI data is still controversial. 
Our findings obtained using GCA still need to be validated with other techniques.

\section{CONCLUSION}

Using resting-state functional and GCAs, we revealed decreased functional connectivity between CI1.R and AG.L, increased functional connectivity between CI2.R and STG.R, and increased mutual effective connectivities between Cl1.R and AG.L in chess experts. These results indicated long-term professional training could modulate the functions of MT area. Our findings also suggested that CI1.R and CI2.R have a critical effect on chess visual practice, and increased functional connections between CI2.R and STG.R may in turn facilitate communication within the network.

\section{DATA AVAILABILITY STATEMENT}

Publicly available datasets were analyzed in this study. This data can be found here: http://fcon_1000.projects.nitrc.org/indi/pro/ wchsu_li_index.html.

\section{REFERENCES}

Albright, T. D. (1984). Direction and orientation selectivity of neurons in visual area MT of the macaque. J. Neurophysiol. 52, 1106-1130. doi: 10.1152/jn.1984. 52.6.1106

Allison, T., Puce, A., and McCarthy, G. (2000). Social perception from visual cues: role of the STS region. Trends Cogn. Sci. 4, 267-278. doi: 10.1016/s13646613(00)01501-1

Amidzic, O., Riehle, H. J., Fehr, T., Wienbruch, C., and Elbert, T. (2001). Pattern of focal gamma-bursts in chess players. Nature 412:603. doi: 10.1038/35088119

Astafiev, S. V., Stanley, C. M., Shulman, G. L., and Corbetta, M. (2004). Extrastriate body area in human occipital cortex responds to the performance of motor actions. Nat. Neurosci. 7, 542-548. doi: 10.1038/nn1241

Bilalic, M., Langner, R., Erb, M., and Grodd, W. (2010). Mechanisms and neural basis of object and pattern recognition: a study with chess experts. J. Exp. Psychol. Gen. 139, 728-742. doi: 10.1037/a0020756

Bilalic, M., Langner, R., Ulrich, R., and Grodd, W. (2011). Many faces of expertise: fusiform face area in chess experts and novices. J. Neurosci. 31, 10206-10214. doi: 10.1523/jneurosci.5727-10.2011

Corbetta, M., and Shulman, G. L. (2002). Control of goal-directed and stimulusdriven attention in the brain. Nat. Rev. Neurosci. 3, 201-215. doi: 10.1038/ nrn755

Draganski, B., Gaser, C., Busch, V., Schuierer, G., Bogdahn, U., and May, A. (2004). Neuroplasticity: changes in grey matter induced by training. Nature 427, 311-312. doi: 10.1038/427311a

Duan, X., He, S., Liao, W., Liang, D., Qiu, L., Wei, L., et al. (2012a). Reduced caudate volume and enhanced striatal-DMN integration in chess experts. Neuroimage 60, 1280-1286. doi: 10.1016/j.neuroimage.2012.01.047

Duan, X., Liao, W., Liang, D., Qiu, L., Gao, Q., Liu, C., et al. (2012b). Large-scale brain networks in board game experts: insights from a domain-related task and task-free resting state. PLoS One 7:e32532. doi: 10.1371/journal.pone.003 2532

Duan, X., Long, Z., Chen, H., Liang, D., Qiu, L., Huang, X., et al. (2014). Functional organization of intrinsic connectivity networks in Chinese-chess experts. Brain Res. 1558, 33-43. doi: 10.1016/j.brainres.2014.02.033

Fattahi, F., Geshani, A., Jafari, Z., Jalaie, S., and Salman Mahini, M. (2015). Auditory memory function in expert chess players. Med. J. Islam Repub. Iran 29:275.

Fox, M. D., Corbetta, M., Snyder, A. Z., Vincent, J. L., and Raichle, M. E. (2006). Spontaneous neuronal activity distinguishes human dorsal and ventral

\section{ETHICS STATEMENT}

The studies involving human participants were reviewed and approved by the local Institutional Review Board of the West China Hospital of Sichuan University. The patients/participants provided their written informed consent to participate in this study.

\section{AUTHOR CONTRIBUTIONS}

LS and PD designed the study. LS, YG, JL, and PD performed the research. LS and JL analyzed the data. LS, YG, and PD wrote the manuscript. All authors contributed to the article and approved the submitted version.

\section{FUNDING}

This work was supported by the Teaching Reform Project of Weifang Medical University (2019YB029).

attention systems. Proc. Natl. Acad. Sci. U S A. 103, 10046-10051. doi: 10.1073/ pnas.0604187103

Gao, J., Zeng, M., Dai, X., Yang, X., Yu, H., Chen, K., et al. (2020). Functional segregation of the middle temporal visual motion area revealed with coactivation-based parcellation. Front. Neurosci. 14:427. doi: 10.3389/fnins. 2020.00427

Gaser, C., and Schlaug, G. (2003a). Brain structures differ between musicians and non-musicians. J. Neurosci. 23, 9240-9245. doi: 10.1523/jneurosci.23-2709240.2003

Gaser, C., and Schlaug, G. (2003b). Gray matter differences between musicians and nonmusicians. Ann. N. Y. Acad. Sci. 999, 514-517. doi: 10.1196/annals. 1284.062

Hampson, M., Tokoglu, F., Sun, Z., Schafer, R. J., Skudlarski, P., Gore, J. C., et al. (2006). Connectivity-behavior analysis reveals that functional connectivity between left BA39 and Broca's area varies with reading ability. Neuroimage 31, 513-519. doi: 10.1016/j.neuroimage.2005.12.040

Hoffman, E. A., and Haxby, J. V. (2000). Distinct representations of eye gaze and identity in the distributed human neural system for face perception. Nat. Neurosci. 3, 80-84.

Horwitz, B., Rumsey, J. M., and Donohue, B. C. (1998). Functional connectivity of the angular gyrus in normal reading and dyslexia. Proc. Natl. Acad. Sci. U S A. 95, 8939-8944. doi: 10.1073/pnas.95.15.8939

Kincade, J. M., Abrams, R. A., Astafiev, S. V., Shulman, G. L., and Corbetta, M. (2005). An event-related functional magnetic resonance imaging study of voluntary and stimulus-driven orienting of attention. J. Neurosci. 25, 45934604. doi: 10.1523/jneurosci.0236-05.2005

Kolster, H., Peeters, R., and Orban, G. A. (2010). The retinotopic organization of the human middle temporal area MT/V5 and its cortical neighbors. J. Neurosci. 30, 9801-9820. doi: 10.1523/jneurosci.2069-10.2010

Krawczyk, D. C., Boggan, A. L., McClelland, M. M., and Bartlett, J. C. (2011). The neural organization of perception in chess experts. Neurosci. Lett. 499, 64-69. doi: 10.1016/j.neulet.2011.05.033

Lewis, C. M., Baldassarre, A., Committeri, G., Romani, G. L., and Corbetta, M. (2009). Learning sculpts the spontaneous activity of the resting human brain. Proc. Natl. Acad. Sci. U S A. 106, 17558-17563. doi: 10.1073/pnas.0902455106

Li, K., Jiang, J., Qiu, L., Yang, X., Huang, X., Lui, S., et al. (2015). A multimodal MRI dataset of professional chess players. Sci. Data 2:150044.

Maguire, E. A., Gadian, D. G., Johnsrude, I. S., Good, C. D., Ashburner, J. Frackowiak, R. S., et al. (2000). Navigation-related structural change in the 
hippocampi of taxi drivers. Proc. Natl. Acad. Sci. U S A. 97, 4398-4403. doi: 10.1073/pnas.070039597

Onofrj, M., Curatola, L., Valentini, G., Antonelli, M., Thomas, A., and Fulgente, T. (1995). Non-dominant dorsal-prefrontal activation during chess problem solution evidenced by single photon emission computerized tomography (SPECT). Neurosci. Lett. 198, 169-172. doi: 10.1016/0304-3940(95)11985-6

Pelphrey, K. A., Morris, J. P., Michelich, C. R., Allison, T., and McCarthy, G. (2005). Functional anatomy of biological motion perception in posterior temporal cortex: an FMRI study of eye, mouth and hand movements. Cereb. Cortex 15, 1866-1876. doi: 10.1093/cercor/bhi064

Poirier, C., Collignon, O., Scheiber, C., Renier, L., Vanlierde, A., Tranduy, D., et al. (2006). Auditory motion perception activates visual motion areas in early blind subjects. NeuroImage 31, 279-285. doi: 10.1016/j.neuroimage.2005.11.036

Power, J. D., Barnes, K. A., Snyder, A. Z., Schlaggar, B. L., and Petersen, S. E. (2012). Spurious but systematic correlations in functional connectivity MRI networks arise from subject motion. NeuroImage 59, 2142-2154. doi: 10.1016/ j.neuroimage.2011.10.018

Rajarethinam, R. P., DeQuardo, J. R., Nalepa, R., and Tandon, R. (2000). Superior temporal gyrus in schizophrenia: a volumetric magnetic resonance imaging study. Schizophr Res. 41, 303-312. doi: 10.1016/s0920-9964(99)00083-3

Seth, A. K. (2010). A MATLAB toolbox for Granger causal connectivity analysis. J. Neurosci. Methods 186, 262-273. doi: 10.1016/j.jneumeth.2009.11.020

Smith, A. T., Wall, M. B., Williams, A. L., and Singh, K. D. (2006). Sensitivity to optic flow in human cortical areas MT and MST. Eur. J. Neurosci. 23, 561-569. doi: 10.1111/j.1460-9568.2005.04526.x

Song, L., Peng, Q., Liu, S., and Wang, J. (2020). Changed hub and functional connectivity patterns of the posterior fusiform gyrus in chess experts. Brain Imag. Behav. 14, 797-805. doi: 10.1007/s11682-018-0020-0

van der Maas, H. L., and Wagenmakers, E. J. (2005). A psychometric analysis of chess expertise. Am. J. Psychol. 118, 29-60.

Wang, J., Fan, L., Wang, Y., Xu, W., Jiang, T., Fox, P. T., et al. (2015). Determination of the posterior boundary of Wernicke's area based on multimodal connectivity profiles. Hum. Brain Mapp. 36, 1908-1924. doi: 10.1002/hbm.22745

Wang, J., Tian, Y., Wang, M., Cao, L., Wu, H., Zhang, Y., et al. (2016). A lateralized top-down network for visuospatial attention and neglect. Brain Imaging Behav. 10, 1029-1037. doi: 10.1007/s11682-015-9460-y

Wang, J., Wei, Q., Bai, T., Zhou, X., Sun, H., Becker, B., et al. (2017a). Electroconvulsive therapy selectively enhanced feedforward connectivity from fusiform face area to amygdala in major depressive disorder. Soc. Cogn. Affect. Neurosci. 12, 1983-1992. doi: 10.1093/scan/nsx100

Wang, J., Xie, S., Guo, X., Becker, B., Fox, P. T., Eickhoff, S. B., et al. (2017b). correspondent functional topography of the human left inferior parietal lobule at rest and under task revealed using resting-state fmri and coactivation based parcellation. Hum. Brain Mapp. 38, 1659-1675. doi: 10.1002/hbm.23488

Wang, L., Wei, Q., Wang, C., Xu, J., Wang, K., Tian, Y., et al. (2019). Altered functional connectivity patterns of insular subregions in major depressive disorder after electroconvulsive therapy. Brain Imaging Behav. 14, 753-761. doi: $10.1007 /$ s1 1682-018-0013-z

Watkins, K. E., Shakespeare, T. J., O’Donoghue, M. C., Alexander, I., Ragge, N., Cowey, A., et al. (2013). Early auditory processing in area V5/MT+ of the congenitally blind brain. J. Neurosci. 33, 18242-18246. doi: 10.1523/jneurosci. 2546-13.2013

Wu, Y., Wang, J., Zhang, Y., Zheng, D., Zhang, J., Rong, M., et al. (2016). The Neuroanatomical Basis for Posterior Superior Parietal Lobule Control Lateralization of Visuospatial Attention. Front. Neuroanat. 10:32. doi: 10.3389/ fnana.2016.00032

Zevin, J. (2009). Word Recognition. Encyclopedia Neurosci. 517-522. doi: 10.1016/ b978-008045046-9.01881-7

Zhou, S. Q., Jin, L. L., He, J. Z., Zeng, Q. R., Wu, Y., Cao, Z. W., et al. (2018). Distributed performance of white matter properties in chess players: a DWI study using automated fiber quantification. Brain Res. 1700, 9-18. doi: 10.1016/ j.brainres.2018.07.003

Zou, L., Ding, G., Abutalebi, J., Shu, H., and Peng, D. (2012). Structural plasticity of the left caudate in bimodal bilinguals. Cortex 48, 1197-1206. doi: 10.1016/j. cortex.2011.05.022

Conflict of Interest: The authors declare that the research was conducted in the absence of any commercial or financial relationships that could be construed as a potential conflict of interest.

Copyright (c) 2020 Song, Ge, Long and Dong. This is an open-access article distributed under the terms of the Creative Commons Attribution License (CC BY). The use, distribution or reproduction in other forums is permitted, provided the original author(s) and the copyright owner(s) are credited and that the original publication in this journal is cited, in accordance with accepted academic practice. No use, distribution or reproduction is permitted which does not comply with these terms. 